\title{
A brief review of genome editing technology for generating animal models
}

\author{
Haoyi WANG $(\bowtie)^{1,2,3}$, Sen WU $(\bowtie)^{4,5}$, Mario R. CAPECCHI $(\bowtie)^{6}$, Rudolf JAENISCH $(\bowtie)^{7,8}$ \\ 1 State Key Laboratory of Stem Cell and Reproductive Biology, Institute of Zoology, Chinese Academy of Sciences, Beijing 100101, China \\ 2 University of the Chinese Academy of Sciences, Beijing 100049, China \\ 3 Institute for Stem Cell and Regeneration, Chinese Academy of Sciences, Beijing 100101, China \\ 4 Beijing Advanced Innovation Center for Food Nutrition and Human Health, China Agricultural University, Beijing 100193, China \\ 5 State Key Laboratory of Agrobiotechnology, College of Biological Sciences, China Agricultural University, Beijing 100193, China \\ 6 Department of Human Genetics, University of Utah School of Medicine, Salt Lake City, UT 84112, USA \\ 7 Whitehead Institute for Biomedical Research, Cambridge, MA 02142, USA \\ 8 Department of Biology, Massachusetts Institute of Technology, Cambridge, MA 02142, USA
}

\begin{abstract}
The recent development of genome editing technologies has given researchers unprecedented power to alter DNA sequences at chosen genomic loci, thereby generating various genetically edited animal models. This mini-review briefly summarizes the development of major genome editing tools, focusing on the application of these tools to generate animal models in multiple species.
\end{abstract}

Keywords animal model, CRISPR, genome editing, TALEN, ZFN

Animal models are invaluable tools for understanding biology and developing therapies for human diseases. The capability of making precise alterations at chosen genomic loci in cells, or the whole animal, has been established and improved upon at an unprecedented speed, thanks to the development of genome editing technology. This minireview briefly introduces the key developments in genome editing technology and its applications to animal model creation. Given that this is a brief review, it is not possible to include all important contributions here, and we apologize to those whose work has been unintentionally omitted.

The capability of precisely editing any position in the genome has long been a dream of biologists. Recombinant DNA technology has enabled scientists to engineer DNA molecules in the test tube with great precision since the 1970s. Transgenes can be expressed by introducing engineered DNA molecules into different cells. These

Received January 1, 2020; accepted January 27, 2020

Correspondences: wanghaoyi@ioz.ac.cn, swu@cau.edu.cn,

mario.capecchi@genetics.utah.edu,

jaenisch@wi.mit.edu
DNA fragments become integrated with low efficiency into the genome of cells, leading to the addition of constructed genetic information, although at random locations. The first demonstration that exogenous DNA could be introduced into early mammalian embryos was made by Jaenisch and Mintz in $1974^{[1]}$, followed by the demonstration of germ line transmission of the exogenous $\mathrm{DNA}^{[2]}$. Subsequent efforts of Gordon et al. showed that linear DNA fragments injected into the pronucleus of mouse embryos can lead to the generation of transgenic animals ${ }^{[3]}$. This technology is still being actively used for expressing exogenous genes in the mouse and other species. While transgenic animals are useful for expressing a particular transgene and to generate insertional mutations ${ }^{[4]}$, integration of the DNA into the genome is random and thus this approach cannot be used to disrupt specific genes.

The seminal work of Smithies et al. ${ }^{[5]}$ and Thomas et al. ${ }^{[6]}$ showed that an exogenous DNA fragment could be precisely integrated into the desired genomic locus via homologous recombination (HR), a cell-intrinsic DNA repair mechanism. Although the efficiency of this genetargeting technology ${ }^{[7]}$ was initially low, the rare clones containing the desired targeting event could be enriched and selected by a variety of elegantly designed strategies, leading to the derivation of clonal cell lines containing the desired genetic modification. The combination of genetargeting and mouse embryonic stem cell (mESC) technologies ${ }^{[8]}$ made it possible to generate a mouse composed entirely of cells containing the designed predetermined genetic modification ${ }^{[9,10]}$. While this approach transformed modern biology and resulted in the award of a Nobel Prize, its application was limited to mice and more recently to rats ${ }^{[11]}$, mainly because embryonic 
stem cells with germ line contribution capability have not been established in most mammalian species. Also, gene targeting in most human cell types is inefficient, thereby limiting its application for genetic correction-based therapy. A means of increasing the efficiency of HR therefore became a key question for improving the capability of modifying genomes of all life forms.

First in yeast and then in mammalian cells, pioneering work on DNA double-stranded break (DSB) repair demonstrated that site-specific DSB in the genome could stimulate the rate of local HR by several orders of magnitude ${ }^{[12,13]}$. Also, it was shown that, once the DSB was introduced, in addition to the HR pathway the nonhomologous end joining (NHEJ) pathway could repair the DSB, often leading to the introduction of small insertions and/or deletions (indels) at target sites. These results motivated the development of methods that generate DNA DSB at a specific locus in the genome. The earlier efforts were aimed at engineering a class of rare-cutting endonuclease, meganucleases, that recognize long stretches of DNA sequences ${ }^{[14]}$. However, it has been quite difficult to engineer naturally occurring meganucleases to bind and cleave chosen DNA target sequences and this has greatly limited their wider application.

Zinc finger proteins (ZFPs) are one of the most abundant types of proteins in eukaryotes and the largest transcription factor family in human ${ }^{[15-17]}$. With the coordination of zinc ions, each zinc finger domain recognizes a 3-4 bp DNA sequence, and a combination of several fingers allows for the recognition of a longer sequence. Zinc finger nuclease (ZFN) is generated by fusing a zinc finger binding domain with the FokI nuclease domain. A pair of ZFNs binds to two proximal sites next to each other, leading to the homodimerization of FokI, generating site-specific DNA DSBs in the genome. ZFNs have been used to generate various indel mutations as well as to stimulate homologydirected repair (HDR) in different species including human cells ${ }^{[18]}$. Upon their injection into the nuclei of Xenopus oocytes, a pair of ZFNs binding in the opposite direction induced DNA cleavage efficiently, with the linker between FokI and ZF domains constraining the spacing of the two binding sites ${ }^{[19]}$. Further, by introducing heat-shock promoter-driven ZFN transgenes into the Drosophila germline, ZFNs efficiently knocked out endogenous genes ${ }^{[20]}$ or increased the frequency of targeted integration of transgenes into the chromosome ${ }^{[21]}$. Several studies in 2008 showed that direct injection of ZFNs into embryos of Drosophila and zebrafish could lead to the efficient generation of gene knockout $(\mathrm{KO})$ animals ${ }^{[22-24]}$. In 2009, Geurts et al. showed that microinjection of ZFN into rat zygotes led to the generation of a gene $\mathrm{KO} \mathrm{rat}^{[25]}$, demonstrating a powerful strategy to generate gene-edited animals without limitation due to HR efficiency or availability of germline contributing embryonic stem cells.

Although a variety of strategies have been established to generate ZFPs with designed specificity, they often require significant expertise and intensive screening. This has limited the wider adoption of ZFN technology within the scientific community. In 2009, two groups independently deciphered the mechanism of transcription activator-like effectors (TALEs) recognizing target DNA sequences and found a remarkably simple modular recognition $\operatorname{code}^{[26,27]}$. Depending on the repeat-variable di-residue (RVD), each module of the highly repetitive TALE DNA binding domain can specifically recognize one single DNA base pair. Based on the RVD-nucleotide association, a TALE DNA binding protein could be generated to recognize a sequence of choice by simply linking different modules together. The TALE nuclease (TALEN) system was readily established by replacing the ZF domain with the TALE DNA binding domain. The principle of TALEN is very similar to ZFN with the only difference being that the DNA binding specificity is determined by the array of TALE motifs. Soon afterwards, TALEN was shown to work efficiently in human cells ${ }^{[28,29]}$. By injecting TALEN into zygotes, animals with specific gene KO were generated in various species including non-human primates $^{[30-34]}$. Co-injection of donor template into zygotes leads to efficient HDR, generating animals with precise nucleotide change and targeted gene integration $^{[35-37]}$. Editing mESCs using TALENs generated the first mouse containing targeted mutations on the $\mathrm{Y}$ chromosome ${ }^{[38]}$. TALEN was also used in livestock with well-established cloning technology to generate specific mutations in somatic cell lines which were then cloned to generate genetically-modified animals ${ }^{[39,40]}$.

Although TALENs were much easier to generate than ZFNs, CRISPR (clustered regularly interspaced short palindromic repeat DNA sequences)-Cas9 readily outperformed all previous tools for generating designed double strand breaks and quickly became the method of choice for genome editing. CRISPR-Cas systems are adaptive immune defense mechanisms protecting against invading nucleic acids such as plasmid and phage infection in a large proportion of bacterial and archaeal species ${ }^{[11]}$. Through decades of work, people dissected the mechanism of the CRISPR-Cas system ${ }^{[42]}$, and the biochemical characterization of the Streptococcus pyogenes and Streptococcus thermophilus CRISPR systems demonstrated that Cas9 is an RNA guided DNA endonuclease that targets specific DNA sequences complementary to the 20 nucleotide sequence residing at the $5^{\prime}$ end of the guide $\mathrm{RNA}^{[43,44]}$. Upon appropriate codon optimization, CRISPR-Cas9 was able to efficiently target specific genomic loci in mammalian cells ${ }^{[45,46]}$. By simply designing a 20 -nucleotide sequence within single guide RNA (sgRNA), the Cas9 nuclease-sgRNA complex can generate DNA DSBs at a specific genomic locus. The ease of use and robust performance of the CRISPR-Cas9 system resulted in its rapid adoption by the scientific community.

The efficiency of the CRISPR-Cas9 system makes it particularly attractive for performing multiplex gene 
editing. By co-expressing multiple sgRNAs, Cas9 can generate DSBs at multiple chosen loci within the same cell $^{[46-48]}$. Following the previous principle established using ZFN and TALEN, injection of CRISPR-Cas9 into the zygote leads to highly efficient generation of genetically-modified animals. Mice carrying multiple gene KOs or precise nucleotide changes can be derived within a month ${ }^{[47,49]}$. A similar approach produced genetically edited animals from ever-increasing numbers of species ${ }^{[50-54]}$. To make the process even simpler, several groups developed the protocols to deliver CRISPR-Cas9 components into rat and mouse zygotes by electroporation, which has been successfully adopted by the field ${ }^{[55-59]}$. In addition to modifying the genome of the zygote, germline modification has also been achieved in mice by genome editing of spermatogonial stem cells. After development to spermatids and injection into oocytes, animals with specific genetic modification were derived ${ }^{[60]}$. By combining the gene editing and haploid stem cell technologies, an artificial sperm strategy was established as an efficient method to generate gene-edited mice ${ }^{[61]}$. Whether these strategies can be transferred to other species remains to be tested.

One of the advantages of the CRISPR-Cas9 system is the great flexibility of repurposing its function. Cas9 has two nuclease domains, each capable of cleaving one of the target DNA strands. When either one of these domains is mutated, the Cas9-sgRNA complex becomes a sequence and strand-specific nickase; and when both nuclease domains are mutated, Cas 9 becomes a programmable DNA binding protein (dCas9) without any endonuclease activity. Guided by sgRNA, dCas9 fused with different effector domains can bind to regulatory elements and regulate transcription, as well as epigenetic modifications ${ }^{[62,63]}$. In particular for genome editing, dCas9 or Cas9 nickase fused with single-stranded DNA deaminase was developed into programmable DNA base editors, which can make specific nucleotide changes without introducing DNA DSB or replying on $\operatorname{HDR}^{[64]}$. To construct cytosine base editors, single-stranded DNA cytosine deaminase was used to mediate $\mathrm{C} \cdot \mathrm{G}$ to $\mathrm{T} \cdot \mathrm{A}$ conversions ${ }^{[65,66]}$; to generate adenine base editors capable of converting $A \cdot T$ to $G \cdot C$, the DNA adenine deaminase was artificially evolved from the bacterial tRNA-specific adenosine deaminase $\operatorname{TadA}^{[67]}$. Following the demonstration of efficient base editing in cultured cells, multiple groups introduced base editors into the embryos of various species and demonstrated efficient generation of base-edited animal models ${ }^{[68-73]}$. These studies showed that, in general, base editing generated animals carrying precise single nucleotide editing more efficiently than previous HDR based genome editing strategies.

Generation of genetically edited animal models is becoming much easier with rapidly-improving genome editing technology. New strategies based on recently developed prime-editing ${ }^{[74]}$ and newly discovered site- specific transposon systems ${ }^{[75,76]}$ promise to make even more sophisticatedly-edited animal models more efficiently in the future.

Compliance with ethics guidelines Haoyi Wang, Sen $\mathrm{Wu}$, Mario R. Capecchi, and Rudolf Jaenisch declare that they have no conflicts of interest or financial conflicts to disclose.

This article is a mini-review and does not contain any studies with human or animal subjects performed by any of the authors.

\section{References}

1. Jaenisch R, Mintz B. Simian virus 40 DNA sequences in DNA of healthy adult mice derived from preimplantation blastocysts injected with viral DNA. Proceedings of the National Academy of Sciences of the United States of America, 1974, 71(4): 1250-1254

2. Jaenisch R. Germ line integration and Mendelian transmission of the exogenous Moloney leukemia virus. Proceedings of the National Academy of Sciences of the United States of America, 1976, 73(4): $1260-1264$

3. Gordon J W, Scangos G A, Plotkin D J, Barbosa J A, Ruddle F H. Genetic transformation of mouse embryos by microinjection of purified DNA. Proceedings of the National Academy of Sciences of the United States of America, 1980, 77(12): 7380-7384

4. Schnieke A, Harbers K, Jaenisch R. Embryonic lethal mutation in mice induced by retrovirus insertion into the $\alpha 1$ (I) collagen gene. Nature, 1983, 304(5924): 315-320

5. Smithies O, Gregg R G, Boggs S S, Koralewski M A, Kucherlapati $\mathrm{R} S$. Insertion of DNA sequences into the human chromosomal $\beta$ globin locus by homologous recombination. Nature, 1985, 317 (6034): 230-234

6. Thomas K R, Folger K R, Capecchi M R. High frequency targeting of genes to specific sites in the mammalian genome. Cell, 1986, 44 (3): 419-428

7. Capecchi M R. Gene targeting in mice: functional analysis of the mammalian genome for the twenty-first century. Nature Reviews: Genetics, 2005, 6(6): 507-512

8. Bradley A, Evans M, Kaufman M H, Robertson E. Formation of germ-line chimaeras from embryo-derived teratocarcinoma cell lines. Nature, 1984, 309(5965): 255-256

9. Thomas K R, Capecchi M R. Site-directed mutagenesis by gene targeting in mouse embryo-derived stem cells. Cell, 1987, 51(3): 503-512

10. Doetschman T, Gregg R G, Maeda N, Hooper M L, Melton D W, Thompson S, Smithies O. Targetted correction of a mutant HPRT gene in mouse embryonic stem cells. Nature, 1987, 330(6148): 576578

11. Buehr M, Meek S, Blair K, Yang J, Ure J, Silva J, McLay R, Hall J, Ying Q L, Smith A. Capture of authentic embryonic stem cells from rat blastocysts. Cell, 2008, 135(7): 1287-1298

12. Rouet $P$, Smih F, Jasin M. Introduction of double-strand breaks into the genome of mouse cells by expression of a rare-cutting endonuclease. Molecular and Cellular Biology, 1994, 14(12): 8096-8106

13. Rudin N, Sugarman E, Haber J E. Genetic and physical analysis of double-strand break repair and recombination in Saccharomyces 
cerevisiae. Genetics, 1989, 122(3): 519-534

14. Silva G, Poirot L, Galetto R, Smith J, Montoya G, Duchateau P, Pâques F. Meganucleases and other tools for targeted genome engineering: perspectives and challenges for gene therapy. Current Gene Therapy, 2011, 11(1): 11-27

15. Miller J, McLachlan A D, Klug A. Repetitive zinc-binding domains in the protein transcription factor IIIA from Xenopus oocytes. EMBO Journal, 1985, 4(6): 1609-1614

16. Najafabadi H S, Mnaimneh S, Schmitges F W, Garton M, Lam K N, Yang A, Albu M, Weirauch M T, Radovani E, Kim P M, Greenblatt J, Frey B J, Hughes T R. C2H2 zinc finger proteins greatly expand the human regulatory lexicon. Nature Biotechnology, 2015, 33(5): $555-562$

17. Takatsuji H. Zinc-finger transcription factors in plants. Cellular and Molecular Life Sciences, 1998, 54(6): 582-596

18. Urnov F D, Rebar E J, Holmes M C, Zhang H S, Gregory P D. Genome editing with engineered zinc finger nucleases. Nature Reviews: Genetics, 2010, 11(9): 636-646

19. Bibikova M, Carroll D, Segal D J, Trautman J K, Smith J, Kim Y G, Chandrasegaran S. Stimulation of homologous recombination through targeted cleavage by chimeric nucleases. Molecular and Cellular Biology, 2001, 21(1): 289-297

20. Bibikova M, Golic M, Golic K G, Carroll D. Targeted chromosomal cleavage and mutagenesis in Drosophila using zinc-finger nucleases. Genetics, 2002, 161(3): 1169-1175

21. Bibikova M, Beumer K, Trautman J K, Carroll D. Enhancing gene targeting with designed zinc finger nucleases. Science, 2003, 300 (5620): 764

22. Beumer K J, Trautman J K, Bozas A, Liu J L, Rutter J, Gall J G, Carroll D. Efficient gene targeting in Drosophila by direct embryo injection with zinc-finger nucleases. Proceedings of the National Academy of Sciences of the United States of America, 2008, 105 (50): 19821-19826

23. Doyon Y, McCammon J M, Miller J C, Faraji F, Ngo C, Katibah G E, Amora R, Hocking T D, Zhang L, Rebar E J, Gregory P D, Urnov F D, Amacher S L. Heritable targeted gene disruption in zebrafish using designed zinc-finger nucleases. Nature Biotechnology, 2008, 26(6): 702-708

24. Meng X, Noyes M B, Zhu L J, Lawson N D, Wolfe S A. Targeted gene inactivation in zebrafish using engineered zinc-finger nucleases. Nature Biotechnology, 2008, 26(6): 695-701

25. Geurts A M, Cost G J, Freyvert Y, Zeitler B, Miller J C, Choi V M, Jenkins S S, Wood A, Cui X, Meng X, Vincent A, Lam S, Michalkiewicz M, Schilling R, Foeckler J, Kalloway S, Weiler H, Ménoret S, Anegon I, Davis G D, Zhang L, Rebar E J, Gregory P D, Urnov F D, Jacob H J, Buelow R. Knockout rats via embryo microinjection of zinc-finger nucleases. Science, 2009, 325(5939): 433

26. Boch J, Scholze H, Schornack S, Landgraf A, Hahn S, Kay S, Lahaye T, Nickstadt A, Bonas U. Breaking the code of DNA binding specificity of TAL-type III effectors. Science, 2009, 326 (5959): 1509-1512

27. Moscou M J, Bogdanove A J. A simple cipher governs DNA recognition by TAL effectors. Science, 2009, 326(5959): 1501

28. Miller J C, Tan S, Qiao G, Barlow K A, Wang J, Xia D F, Meng X, Paschon D E, Leung E, Hinkley S J, Dulay G P, Hua K L,
Ankoudinova I, Cost G J, Urnov F D, Zhang H S, Holmes M C, Zhang L, Gregory P D, Rebar E J. A TALE nuclease architecture for efficient genome editing. Nature Biotechnology, 2011, 29(2): 143148

29. Hockemeyer D, Wang H, Kiani S, Lai C S, Gao Q, Cassady J P, Cost G J, Zhang L, Santiago Y, Miller J C, Zeitler B, Cherone J M, Meng X, Hinkley S J, Rebar E J, Gregory P D, Urnov F D, Jaenisch R. Genetic engineering of human pluripotent cells using TALE nucleases. Nature Biotechnology, 2011, 29(8): 731-734

30. Tesson L, Usal C, Ménoret S, Leung E, Niles B J, Remy S, Santiago Y, Vincent A I, Meng X, Zhang L, Gregory P D, Anegon I, Cost G J. Knockout rats generated by embryo microinjection of TALENs. Nature Biotechnology, 2011, 29(8): 695-696

31. Sander J D, Cade L, Khayter C, Reyon D, Peterson R T, Joung J K, Yeh J R J. Targeted gene disruption in somatic zebrafish cells using engineered TALENs. Nature Biotechnology, 2011, 29(8): 697-698

32. Huang P, Xiao A, Zhou M, Zhu Z, Lin S, Zhang B. Heritable gene targeting in zebrafish using customized TALENs. Nature Biotechnology, 2011, 29(8): 699-700

33. Qiu Z, Liu M, Chen Z, Shao Y, Pan H, Wei G, Yu C, Zhang L, Li X, Wang P, Fan H Y, Du B, Liu B, Liu M, Li D. High-efficiency and heritable gene targeting in mouse by transcription activator-like effector nucleases. Nucleic Acids Research, 2013, 41(11): e120

34. Liu H, Chen Y, Niu Y, Zhang K, Kang Y, Ge W, Liu X, Zhao E, Wang C, Lin S, Jing B, Si C, Lin Q, Chen X, Lin H, Pu X, Wang Y, Qin B, Wang F, Wang H, Si W, Zhou J, Tan T, Li T, Ji S, Xue Z, Luo Y, Cheng L, Zhou Q, Li S, Sun Y E, Ji W. TALEN-mediated gene mutagenesis in rhesus and cynomolgus monkeys. Cell Stem Cell, 2014, 14(3): 323-328

35. Remy S, Tesson L, Menoret S, Usal C, De Cian A, Thepenier V, Thinard R, Baron D, Charpentier M, Renaud J B, Buelow R, Cost G J, Giovannangeli C, Fraichard A, Concordet J P, Anegon I. Efficient gene targeting by homology-directed repair in rat zygotes using TALE nucleases. Genome Research, 2014, 24(8): 1371-1383

36. Wefers B, Meyer M, Ortiz O, Hrabé de Angelis M, Hansen J, Wurst W, Kühn R. Direct production of mouse disease models by embryo microinjection of TALENs and oligodeoxynucleotides. Proceedings of the National Academy of Sciences of the United States of America, 2013, 110(10): 3782-3787

37. Bedell V M, Wang Y, Campbell J M, Poshusta T L, Starker C G, Krug R G 2nd, Tan W, Penheiter S G, Ma A C, Leung A Y H, Fahrenkrug S C, Carlson D F, Voytas D F, Clark K J, Essner J J, Ekker S C. In vivo genome editing using a high-efficiency TALEN system. Nature, 2012, 491(7422): 114-118

38. Wang H, Hu Y C, Markoulaki S, Welstead G G, Cheng A W, Shivalila C S, Pyntikova T, Dadon D B, Voytas D F, Bogdanove A J, Page D C, Jaenisch R. TALEN-mediated editing of the mouse Y chromosome. Nature Biotechnology, 2013, 31(6): 530-532

39. Tan W, Carlson D F, Lancto C A, Garbe J R, Webster D A, Hackett $\mathrm{P}$ B, Fahrenkrug S C. Efficient nonmeiotic allele introgression in livestock using custom endonucleases. Proceedings of the National Academy of Sciences of the United States of America, 2013, 110(41): 16526-16531

40. Carlson D F, Tan W, Lillico S G, Stverakova D, Proudfoot C, Christian M, Voytas D F, Long C R, Whitelaw C B, Fahrenkrug S C. Efficient TALEN-mediated gene knockout in livestock. Proceedings 
of the National Academy of Sciences of the United States of America, 2012, 109(43): 17382-17387

41. Makarova K S, Wolf Y I, Alkhnbashi O S, Costa F, Shah S A, Saunders S J, Barrangou R, Brouns S J, Charpentier E, Haft D H, Horvath P, Moineau S, Mojica F J, Terns R M, Terns M P, White M F, Yakunin A F, Garrett R A, van der Oost J, Backofen R, Koonin E V. An updated evolutionary classification of CRISPR-Cas systems. Nature Reviews: Microbiology, 2015, 13(11): 722-736

42. Jiang F, Doudna J A. CRISPR-Cas9 structures and mechanisms. Annual Review of Biophysics, 2017, 46(1): 505-529

43. Gasiunas G, Barrangou R, Horvath P, Siksnys V. Cas9-crRNA ribonucleoprotein complex mediates specific DNA cleavage for adaptive immunity in bacteria. Proceedings of the National Academy of Sciences of the United States of America, 2012, 109 (39): E2579-E2586

44. Jinek M, Chylinski K, Fonfara I, Hauer M, Doudna J A, Charpentier E. A programmable dual-RNA-guided DNA endonuclease in adaptive bacterial immunity. Science, 2012, 337(6096): 816-821

45. Mali P, Yang L, Esvelt K M, Aach J, Guell M, DiCarlo J E, Norville J E, Church G M. RNA-guided human genome engineering via Cas9. Science, 2013, 339(6121): 823-826

46. Cong L, Ran F A, Cox D, Lin S, Barretto R, Habib N, Hsu P D, Wu X, Jiang W, Marraffini L A, Zhang F. Multiplex genome engineering using CRISPR/Cas systems. Science, 2013, 339 (6121): 819-823

47. Wang H, Yang H, Shivalila C S, Dawlaty M M, Cheng A W, Zhang $\mathrm{F}$, Jaenisch R. One-step generation of mice carrying mutations in multiple genes by CRISPR/Cas-mediated genome engineering. Cell, 2013, 153(4): 910-918

48. Yang L, Güell M, Niu D, George H, Lesha E, Grishin D, Aach J, Shrock E, Xu W, Poci J, Cortazio R, Wilkinson R A, Fishman J A, Church G. Genome-wide inactivation of porcine endogenous retroviruses (PERVs). Science, 2015, 350(6264): 1101-1104

49. Yang H, Wang H, Shivalila C S, Cheng A W, Shi L, Jaenisch R. One-step generation of mice carrying reporter and conditional alleles by CRISPR/Cas-mediated genome engineering. Cell, 2013, 154(6): 1370-1379

50. Li W, Teng F, Li T, Zhou Q. Simultaneous generation and germline transmission of multiple gene mutations in rat using CRISPR-Cas systems. Nature Biotechnology, 2013, 31(8): 684-686

51. Li D, Qiu Z, Shao Y, Chen Y, Guan Y, Liu M, Li Y, Gao N, Wang L, Lu X, Zhao Y, Liu M. Heritable gene targeting in the mouse and rat using a CRISPR-Cas system. Nature Biotechnology, 2013, 31(8): 681-683

52. Hwang W Y, Fu Y, Reyon D, Maeder M L, Tsai S Q, Sander J D, Peterson R T, Yeh J R, Joung J K. Efficient genome editing in zebrafish using a CRISPR-Cas system. Nature Biotechnology, 2013, 31(3): 227-229

53. Xiang G, Ren J, Hai T, Fu R, Yu D, Wang J, Li W, Wang H, Zhou Q. Editing porcine IGF2 regulatory element improved meat production in Chinese Bama pigs. Cellular and Molecular Life Sciences, 2018, 75(24): 4619-4628

54. Niu Y, Shen B, Cui Y, Chen Y, Wang J, Wang L, Kang Y, Zhao X, Si W, Li W, Xiang A P, Zhou J, Guo X, Bi Y, Si C, Hu B, Dong G, Wang H, Zhou Z, Li T, Tan T, Pu X, Wang F, Ji S, Zhou Q, Huang $\mathrm{X}$, Ji W, Sha J. Generation of gene-modified cynomolgus monkey via Cas9/RNA-mediated gene targeting in one-cell embryos. Cell, 2014, 156(4): 836-843

55. Hashimoto M, Takemoto T. Electroporation enables the efficient mRNA delivery into the mouse zygotes and facilitates CRISPR/ Cas9-based genome editing. Scientific Reports, 2015, 5(1): 11315

56. Qin W, Dion S L, Kutny P M, Zhang Y, Cheng A W, Jillette N L, Malhotra A, Geurts A M, Chen Y G, Wang H. Efficient CRISPR/ Cas9-mediated genome editing in mice by zygote electroporation of nuclease. Genetics, 2015, 200(2): 423-430

57. Kaneko T, Sakuma T, Yamamoto T, Mashimo T. Simple knockout by electroporation of engineered endonucleases into intact rat embryos. Scientific Reports, 2014, 4(1): 6382

58. Wang W, Kutny P M, Byers S L, Longstaff C J, DaCosta M J, Pang C, Zhang Y, Taft R A, Buaas F W, Wang H. Delivery of Cas9 protein into mouse zygotes through a series of electroporation dramatically increases the efficiency of model creation. Journal of Genetics and Genomics, 2016, 43(5): 319-327

59. Takahashi G, Gurumurthy C B, Wada K, Miura H, Sato M, Ohtsuka M. GONAD: genome-editing via Oviductal Nucleic Acids Delivery system: a novel microinjection independent genome engineering method in mice. Scientific Reports, 2015, 5(1): 11406

60. Wu Y, Zhou H, Fan X, Zhang Y, Zhang M, Wang Y, Xie Z, Bai M, Yin Q, Liang D, Tang W, Liao J, Zhou C, Liu W, Zhu P, Guo H, Pan $\mathrm{H}, \mathrm{Wu} \mathrm{C}$, Shi H, Wu L, Tang F, Li J. Correction of a genetic disease by CRISPR-Cas9-mediated gene editing in mouse spermatogonial stem cells. Cell Research, 2015, 25(1): 67-79

61. Wei L, Wang X, Yang S, Yuan W, Li J. Efficient generation of the mouse model with a defined point mutation through haploid cellmediated gene editing. Journal of Genetics and Genomics, 2017, 44 (9): 461-463

62. Adli M. The CRISPR tool kit for genome editing and beyond. Nature Communications, 2018, 9(1): 1911

63. Wang H, La Russa M, Qi L S. CRISPR/Cas9 in genome editing and beyond. Annual Review of Biochemistry, 2016, 85(1): 227-264

64. Rees H A, Liu D R. Base editing: precision chemistry on the genome and transcriptome of living cells. Nature Reviews: Genetics, 2018, 19(12): 770-788

65. Nishida K, Arazoe T, Yachie N, Banno S, Kakimoto M, Tabata M, Mochizuki M, Miyabe A, Araki M, Hara K Y, Shimatani Z, Kondo A. Targeted nucleotide editing using hybrid prokaryotic and vertebrate adaptive immune systems. Science, 2016, 353(6305): aaf8729

66. Komor A C, Kim Y B, Packer M S, Zuris J A, Liu D R. Programmable editing of a target base in genomic DNA without double-stranded DNA cleavage. Nature, 2016, 533(7603): 420-424

67. Gaudelli N M, Komor A C, Rees H A, Packer M S, Badran A H, Bryson D I, Liu D R. Programmable base editing of A $\cdot T$ to $G \cdot C$ in genomic DNA without DNA cleavage. Nature, 2017, 551(7681): 464-471

68. Liu Z, Lu Z, Yang G, Huang S, Li G, Feng S, Liu Y, Li J, Yu W, Zhang Y, Chen J, Sun Q, Huang X. Efficient generation of mouse models of human diseases via ABE- and BE-mediated base editing. Nature Communications, 2018, 9(1): 2338

69. Liang P, Sun H, Sun Y, Zhang X, Xie X, Zhang J, Zhang Z, Chen Y, Ding C, Xiong Y, Ma W, Liu D, Huang J, Songyang Z. Effective gene editing by high-fidelity base editor 2 in mouse zygotes. Protein 
\& Cell, 2017, 8(8): 601-611

70. Kim K, Ryu S M, Kim S T, Baek G, Kim D, Lim K, Chung E, Kim S, Kim J S. Highly efficient RNA-guided base editing in mouse embryos. Nature Biotechnology, 2017, 35(5): 435-437

71. Ma Y, Yu L, Zhang X, Xin C, Huang S, Bai L, Chen W, Gao R, Li J, Pan S, Qi X, Huang X, Zhang L. Highly efficient and precise base editing by engineered dCas9-guide tRNA adenosine deaminase in rats. Cell Discovery, 2018, 4(1): 39

72. Liu Z, Chen M, Chen S, Deng J, Song Y, Lai L, Li Z. Highly efficient RNA-guided base editing in rabbit. Nature Communications, 2018, 9(1): 2717

73. Yang L, Zhang X, Wang L, Yin S, Zhu B, Xie L, Duan Q, Hu H, Zheng R, Wei Y, Peng L, Han H, Zhang J, Qiu W, Geng H, Siwko S,
Zhang X, Liu M, Li D. Increasing targeting scope of adenosine base editors in mouse and rat embryos through fusion of TadA deaminase with Cas9 variants. Protein \& Cell, 2018, 9(9): 814-819

74. Anzalone A V, Randolph P B, Davis J R, Sousa A A, Koblan L W, Levy J M, Chen P J, Wilson C, Newby G A, Raguram A, Liu D R. Search-and-replace genome editing without double-strand breaks or donor DNA. Nature, 2019, 576(7785): 149-157

75. Klompe S E, Vo P L H, Halpin-Healy T S, Sternberg S H. Transposon-encoded CRISPR-Cas systems direct RNA-guided DNA integration. Nature, 2019, 571(7764): 219-225

76. Strecker J, Ladha A, Gardner Z, Schmid-Burgk J L, Makarova K S, Koonin E V, Zhang F. RNA-guided DNA insertion with CRISPRassociated transposases. Science, 2019, 365(6448): 48-53 